\title{
Monitoramento de áreas embargadas por desmatamento ilegal
}

\author{
Monitoring embargoed areas by illegal deforestation \\ Douglas Rafael Vidal de Moraes ${ }^{1}$ \\ Luiz Cortinhas Ferreira Neto ${ }^{2}$ \\ Merilene do Socorro Silva Costa ${ }^{3}$ \\ Aline Maria Meiguins de Lima ${ }^{4}$ \\ Ima Célia Guimarães Vieira ${ }^{5}$ \\ Jugurta Lisboa Filho ${ }^{6}$ \\ Marcos Adami 7
}

Recebido em abril de 2018.

Aprovado em novembro de 2018.

\begin{abstract}
RESUMO
A Floresta Amazônica abrange 1/3 das florestas tropicais úmidas do planeta. Desta área, aproximadamente $62 \%$ encontra-se no território brasileiro sendo o mais extenso dos biomas brasileiros predominantemente florestais. Considerando as dimensões continentais da Amazônia brasileira e, portanto, todas as dificuldades que evidentemente se apresentam ao policiamento e fiscalização de toda a sua extensão, o monitoramento in loco de todas as áreas autuadas nas ações de fiscalização de combate ao desmatamento torna-se algo quase que inexecutável. O IBAMA é o órgão responsável por essa hercúlea tarefa, de verificar se as leis ambientais estão sendo obedecidas. Caso exista alguma infração, este instituto pode embargar e multar os proprietários. Entretanto, torna-se necessário avaliar se os embargos estão sendo respeitados. Portanto, este estudo teve o objetivo de verificar a efetividade dos embargos em áreas atuadas por desmatamento ilegal, no período de 2004 a 2016, no sudeste do Estado do Pará. Para isto desenvolveu-se uma aplicação web para o monitoramento dessas áreas, tendo como suporte técnicas de geoprocessamento e sensoriamento remoto. A análise destas áreas foi realizada pela interpretação do perfil temporal do NDVI-MODIS juntamente com imagens Landsat 5 e 8. Como resultado,
\end{abstract}

\footnotetext{
${ }^{1}$ Programa de Pós-graduação em Ciência da Computação, Universidade Federal de Viçosa, Brasil. Email: moraesdrv@gmail.com

${ }^{2}$ Programa de Pós-graduação em Engenharia Elétrica, Universidade Federal do Pará, Brasil. E-mail: luizcf14@gmail.com

${ }^{3}$ Professora em Universidade Federal Rural da Amazônia, Brasil. E-mail: merilene.costa@ufra.edu.br 4Professora em Programa de Pós-graduação em Ciências Ambientais, Universidade Federal do Pará, Brasil. E-mail: ameiguins@ufpa.br

5Pesquisadora em Museu Paraense Emílio Goeldi, Brasil. E-mail: ima@museu-goeldi.br

'Professor em Programa de Pós-graduação em Ciência da Computação. Universidade Federal de Viçosa. Brasil. E-mail: jugurta@ufv.br

${ }^{7}$ Pesquisador em Instituto Nacional de Pesquisas Espaciais, Brasil. E-mail: marcos.adami@inpe.br
}

Rev. Bras. de Cartografia, vol. 70, Edição Especial "XXVII Congresso Brasileiro de 
pode-se observar que em apenas $30 \%$ dos polígonos houve o cumprimento do embargo (regeneração natural), enquanto os outros $70 \%$ estavam irregulares, onde foi encontrado classes de agricultura ou pasto, caracterizando descumprimento de embargo. Os resultados indicaram um elevado potencial da ferramenta para o monitoramento e apresentando-se como excelente recurso de suporte para a fiscalização ambiental.

PALAVRAS-CHAVE: Amazônia, Índice de vegetação, Fiscalização ambiental.

\begin{abstract}
The Amazon Rainforest covers The Amazon Forest covers one third of all the humid tropical rainforests of the planet and approximately $62 \%$ of this area is founded in Brazilian territory, being the most extensive Brazilian biome. Thus, considering the continental dimensions of the Brazilian Amazon and therefore all the obstacles faced when it concerns to the policing and reinforcement of its whole extension, it is evident that monitoring in loco all the areas that have been fined in the reinforcement actions to fight against deforestation becomes almost an impracticable task. The IBAMA is the public organ that is responsible for this herculean task: verifying if the environmental laws are being obeyed. If there is an infraction, this institute may promote embargoes and fine the owners. However, it is necessary to assess whether the embargoes are being respected or not. Therefore, this study aimed verifying the effectiveness of the embargoes in areas affected by illegal deforestation, from 2004 to 2016, situated in the southeast of the State of Pará. It consists out in a web application to monitor these areas, supported by geoprocessing and remote sensing techniques. The analysis of these areas was performed by interpreting the temporal profile of NDVI-MODIS together with Landsat5 and Landsat8 images. As a result, it could be observed that only $30 \%$ of the polygons, the embargoes were observed (natural regeneration). The results indicated a high potential of the tool for monitoring, which presented itself as an excellent support resource for environmental monitoring.
\end{abstract}

KEYWORDS: Amazon, Vegetation Index, Environmental Inspection

\title{
Introdução
}

A Floresta Amazônica abrange 1/3 das florestas tropicais úmidas do planeta (BECKER, 2005; THERY, 2005). Desta área, aproximadamente 62\% encontra-se no território brasileiro sendo o mais extenso dos biomas brasileiros predominantemente florestais abrangendo integralmente os estados do Acre, Amapá, Amazonas, Pará, Rondônia, Roraima e ainda uma parte do Maranhão, Tocantins e Mato Grosso (IBGE, 2015). Este bioma é muito biodiverso e abriga um grande número de espécies vegetais e animais, 
muitas delas endêmicas (MITTERMEIER et al., 2005) e o intenso processo de desflorestamento que impera nesta região desde a década de 1960 é considerado um grande problema ambiental (DINIZ et al., 2015; LUI e MOLINA, 2016; KALAMANDEEN, et al., 2018; VEIGA e EHLERS, 2010).

$\mathrm{Na}$ década atual o ritmo do desmatamento diminuiu de $27,8 \mathrm{mil} \mathrm{\textrm {km } ^ { 2 }}$ em 2004 para 4,6 mil km² em 2012, o menor valor de toda a série histórica. O último dado do Projeto de Monitoramento do Desmatamento na Amazônia Legal por Satélite (PRODES) do Instituto Nacional de Pesquisas Espaciais (INPE), aponta que, em 2016, foram desmatados $7.893 \mathrm{~km}^{2}$ na Amazônia Legal (PRODES, 2017), representando um aumento de $27 \%$ em relação à taxa do ano anterior. Esta redução, quando comparada a 2004, se deu em função do Plano de Ação para Prevenção e Controle do Desmatamento na Amazônia Legal (PPCDAm), principalmente devido ao eixo de monitoramento e controle ambiental (IPEA, 2011).

Assim, destaca-se a importância de ações continuadas que permitam uma avaliação espaço temporal da evolução do desmatamento na Amazônia e que tragam informações que direcionem os planos de desenvolvimento e políticas na região, com vistas a promoção do uso mais sustentável do território. Entretanto, considerando as dimensões continentais da Amazônia brasileira e, portanto, todas as dificuldades que evidentemente se apresentam ao policiamento e fiscalização de toda a sua extensão, o monitoramento in loco de todas as áreas nas ações de fiscalização de combate ao desmatamento torna-se uma tarefa hercúlea. Este estudo tem por objetivo analisar o cumprimento das ordens de embargo das áreas autuadas no período de 2004 a 2016 nos municípios de Dom Eliseu, Paragominas, Rondon do Pará e Ulianópolis, no estado do Pará. Assim, pretende-se auxiliar o monitoramento das áreas embargadas pelo IBAMA por desmatamento ilegal de forma mais ágil. 


\section{Políticas Públicas de Prevenção e Controle do Desmatamento Ilegal}

O monitoramento e a repressão são, atualmente, as estratégias principais para combater o desflorestamento no âmbito da legislação e políticas ambiental vigentes (CASTELO, 2015; BIZZO e FARIAS, 2017).

Desde o ano de 1934, o Brasil conta com leis e/ou decretos de cunho ambiental com o objetivo de definir as formas de utilização das terras no território brasileiro, onde estas podem ser exploradas para diferentes tipos de produção rural ou preservadas (SILVA, 2011; BRASIL, 2012).

Devido as altas e crescentes taxas de desflorestamento que vinham acontecendo, em 2004, foi criado o Plano de Ação para Prevenção e Controle do Desmatamento na Amazônia Legal (PPCDAm) com o objetivo de reduzir de forma contínua e consistente o desmatamento e criar condições para se estabelecer um modelo de desenvolvimento sustentável na Amazônia Legal. Um dos principais desafios iniciais foi integrar o combate ao desmatamento às políticas de Estado, partindo-se do princípio de que o combate às causas do desmatamento não poderia mais ser conduzido de forma isolada pelos órgãos ambientais. A execução do PPCDAm conta com diversas ações articuladas em torno dos quatro eixos temáticos, dos quais, para este trabalho destaca-se o eixo Monitoramento e Controle Ambiental (BRASIL, 2004).

\subsection{Monitoramento do desmatamento da floresta amazônica}

Foi na década de 1970 que iniciaram os primeiros levantamentos do desmatamento na Amazônia pelo INPE (VALERIANO et al., 2012). Em 1988, o governo brasileiro solicitou ao INPE desenvolver e operar um sistema de monitoramento anual do desmatamento da Amazônia.

O Decreto 6.321, de 21/12/2007, institui regras de acesso a créditos federais e impõem medidas de regularização fundiária e de redução de desmatamento para o restauro do acesso às linhas de crédito embargadas. $\mathrm{O}$ Art. $2^{\circ}$ torna o INPE responsável pela identificação das áreas, a partir da

Rev. Bras. de Cartografia, vol. 70, Edição Especial "XXVII Congresso Brasileiro de 
dinâmica histórica de desmatamento, com o PRODES, e o Ministério do Meio Ambiente (MMA), com base nesses dados, deve editar anualmente a portaria com a lista de municípios a serem considerados prioritários para ações de prevenção e controle do desmatamento na Amazônia (BRASIL, 2007).

A partir de dados gerados com o PRODES, passaram a serem conhecidas as taxas anuais do desmatamento por corte raso da floresta Amazônica, ou seja, quando havia conversão de floresta nativa para fins agrícolas e para o estabelecimento da atividade pecuária (PIONTEKOWSKI et al., 2014; ALMEIDA et al., 2016).

Outro projeto do INPE para monitoramento da Amazônia é o sistema de Deteç̧ão de Desmatamento em Tempo Real (DETER), utilizado desde 2004, e criado para atender uma demanda do PPCDAm, mapeia usando imagens do sensor Moderate Resolution Imaging Spectroradiometer (MODIS), de 250m de resolução espacial, explorando sua resolução temporal, quase que diária, detectando polígonos de desmatamentos com área maior que 25ha (ANDERSON et al., 2005).

Para acompanhar o novo padrão de dimensão dos polígonos de desmatamento, cujas maiores frequências são de polígonos pequenos, com área menor que 25 ha (VALERIANO et al., 2012), foi criado outro sistema de alertas, chamado de Sistema Detecção de Desmatamento em Tempo Quase Real (DETER-B). Esse sistema utiliza dados dos sensores Advanced Wide Field Sensor (AWiFS) com resolução espacial de 56m, abordo do satélite RESOURCESAT e do Wide Field Imager (WFI), do China - Brazil Earth Resources Satellite (CBERS) com resolução espacial de $64 \mathrm{~m}$. Os dois sistemas de alerta enviam seus dados gerados ao Instituto Brasileiro do Meio Ambiente e dos Recursos Naturais Renováveis (IBAMA), que é responsável pela vigilância e controle do desmatamento (DINIZ et al., 2015).

Esses sistemas de monitoramento fazem parte do eixo de monitoramento e controle do PPCDAm e são utilizados pelo IBAMA nas operações de combate ao desflorestamento. Os dados gerados por esses 
sistemas fornecem subsídios para detectar infrações ambientais por meio de alertas e relatórios, que subsidiam a fiscalização ambiental in loco.

\subsection{Autos de infrações e embargos}

O auto de infração é o documento que abre o processo administrativo destinado à apuração da existência, ou não, da infração ambiental. As infrações ambientais são apuradas mediante processo administrativo específico, no qual é assegurado, ao acusado, o direito constitucional da ampla defesa e do contraditório. Portanto, deve, obrigatoriamente, preencher os requisitos previstos na norma ambiental aplicável (MATOS, 2016).

A base legal que rege as infrações e penalidades referentes ao meio ambiente na esfera da administração é o Decreto $\mathrm{n}^{\circ}$ 6.514, de 22 de julho de 2008. Nele fica estabelecido que o auto de infração deverá ser lavrado pela autoridade ambiental que a houver constatado no local em que foi verificada a infração, e deve conter informações como nome do infrator, endereço e outros dados de identificação civil. Também deve descrever a infração e mencionar o dispositivo legal que oficializa a infração ambiental. $\mathrm{O}$ autuado também receberá sansões, como advertência, multas, apreensões de equipamentos, embargo da área, dentre outros (BRASIL, 2008).

O embargo da área aplicado depois de constatada a infração ambiental pelo agente autuante, no uso do seu poder de polícia, é mencionado no $\S 1^{\circ}$ do art. 101 do decreto 6.514/2008, onde diz que o objetivo é prevenir a ocorrência de novas infrações, resguardar a recuperação ambiental e garantir o resultado prático do processo administrativo (BRASIL, 2008). Desta forma, o embargo vem como uma sanção aplicada pela administração pública, através do órgão ambiental e tem por objetivo propiciar a regeneração do meio ambiente e dar viabilidade à recuperação da área degradada.

O fim do embargo e das penalidades dependerá de decisão da autoridade ambiental, após a apresentação, por parte do autuado, de documentação que regularize a obra ou atividade. Em outros casos, até que a 
área danificada seja recuperada por completo. Portanto a área desmatada deverá ficar sem atividade, aguardando a regeneração natural.

No caso de descumprimento parcial ou total do embargo, está disposto no art. 18 , do decreto $\mathrm{n}^{0} 6.514$, que cabe ao órgão ambiental a aplicação cumulativa das penalidades de suspensão da atividade que originou a infração e da venda de produtos ou subprodutos criados ou produzidos na área objeto do embargo infringido; cancelamento de registros, licenças ou autorizações de funcionamento da atividade econômica junto aos órgãos ambientais e de fiscalização. Além disto, de acordo com o art. 79, do mesmo decreto, o autuado por descumprimento de embargo poderá ser multado em quantia que varia entre $R \$ 10.000,00$ (dez mil reais) a $R \$ 1.000 .000,00$ (um milhão de reais) (BRASIL, 2008).

Neste contexto, pode-se compreender a necessidade de uma ferramenta que ajude no monitoramento ambiental, haja vista a dimensão continental da Amazônia e as dificuldades de acesso a algumas das áreas embargadas por infrações ambientais, que levam à interdição de uma propriedade pública ou privada, à aplicação de multas, e suspensão e cancelamento de atividades, registros, licenças ou autorizações de funcionamento da atividade econômica (BRASIL, 2008).

\section{Aplicação Web para Monitoramento Ambiental}

Considerando a evolução tecnológica e facilidade de acesso, já é possível utilizar em um navegador web, várias das funcionalidades que antes só eram disponíveis em Sistema de Informação Geográfica (SIG), na máquina local (desktop), permitindo, por exemplo, que o usuário utilize uma base geográfica em uma página na internet, para análise multitemporal de uso e cobertura do solo (FREITAS et al., 2011).

Destaca-se que a evolução da internet revolucionou a maneira de disponibilização e acesso aos dados (CABRAL, 2008; ESPÍNDOLA, OLIVEIRA e FORMIGA, 2011). Ela encurtou distâncias e abriu o acesso 
instantâneo a milhões de fontes de conhecimento. Possibilitou também a disseminação de resultados de análise geográfica e informações espaciais para um número bem maior de pessoas. O usuário pode agora acessar, explorar e relacionar informações espaciais através de um navegador em casa, por exemplo. Em decorrência disto, os usuários podem conduzir pesquisas e análises de objetos espaciais de forma online, sem a necessidade de comprar um SIG de preço elevado (FREITAS et al., 2011).

Panazzolo et al., (2014), reforçam a ideia de que sistemas de SIG-Web com ênfase na consulta e disponibilização de dados espaciais na internet têm representado um avanço no acesso às informações georreferenciadas, assim como sua disponibilização na internet para um rápido acesso desde qualquer ponto do país.

Portanto, a criação e aperfeiçoamento de ferramentas que facilitem a análise de dados espaciais na web são fundamentais para pesquisas, principalmente as relacionadas ao meio ambiente.

Como citado anteriormente, o IBAMA é um órgão responsável por identificar a área desmatada ilegalmente e aplicar multas ao proprietário. Assim, quando uma área é embargada por ação do desmatamento ilegal, a área deve sofrer um processo de regeneração, devendo ao proprietário o zelo para que esta área retorne às condições próximas aos originais da floresta. Nesse contexto, as técnicas de sensoriamento remoto e geoprocessamento são opções de ferramentas fundamentais para auxiliar na detecção de áreas desmatadas (SANTOS, MALDONADO e GRAÇA, 2005; LIMANA, 2014).

\section{Material e métodos}

3.1 Área de estudo

A área de estudo deste trabalho são os polígonos embargados por desmatamento, no período de 2004 a 2016, nos municípios de Dom Eliseu, 
Paragominas, Rondon do Pará e Ulianópolis, situados na mesorregião sudeste paraense, microrregião de Paragominas (Figura 1).

A mesorregião sudeste do Pará possui 1,6 milhões de habitantes, correspondendo à terceira mesorregião com maior população, ficando atrás apenas da região metropolitana de Belém e do nordeste paraense (IPEA, 2010). As principais atividades econômicas desenvolvidas nessa mesorregião são a criação bovina, exploração madeireira e, mais recentemente, a produção de grãos, como a soja (NEVES et al., 2014).

Figura 1 - Localização da área de estudo

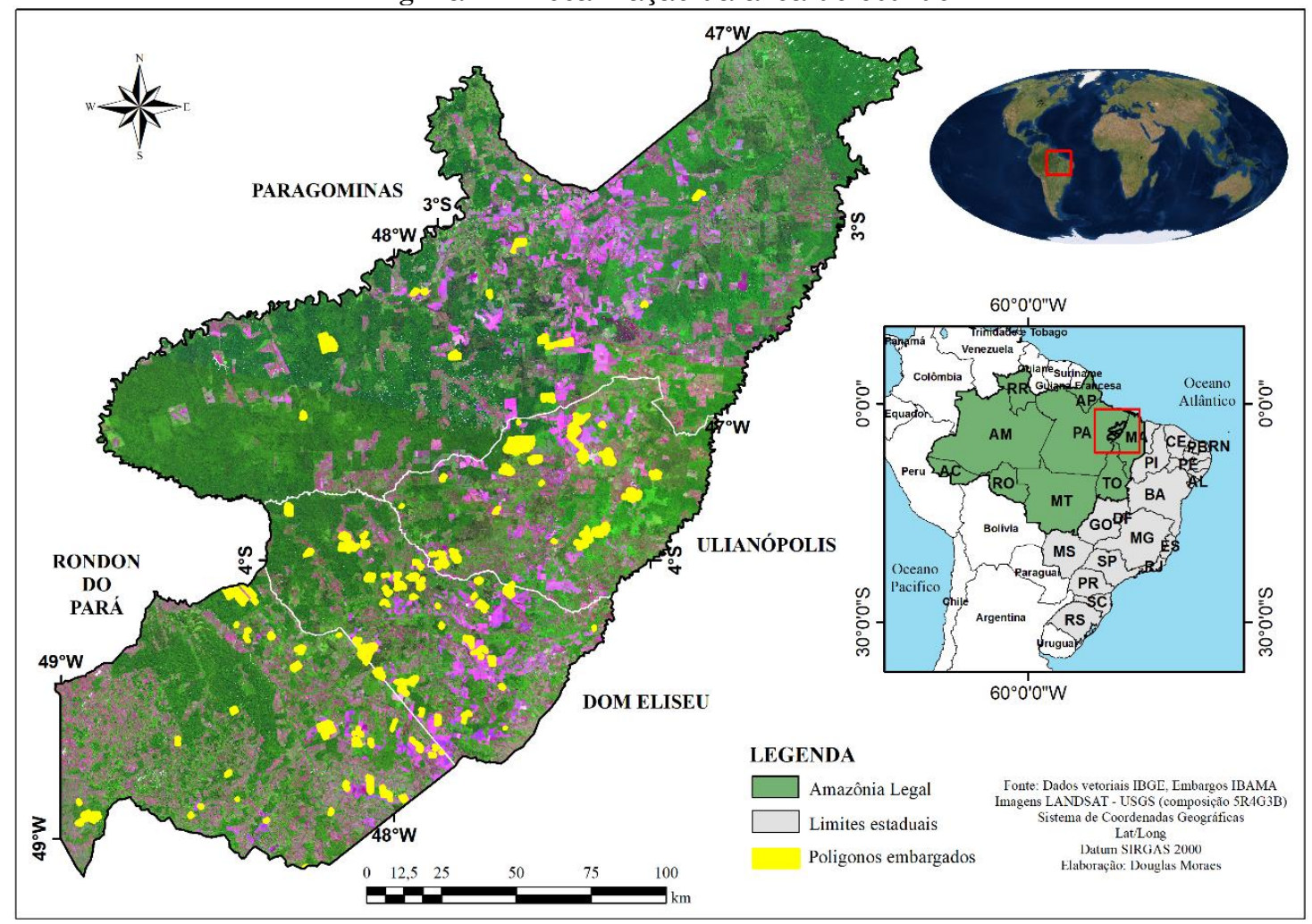

Fonte: Elaborada pelos autores.

\subsection{Material}

Para elaboração deste trabalho foram utilizados os seguintes materiais: 
a) 146 polígonos de áreas embargadas por desmatamento ilegal distribuídos nos municípios de Dom Eliseu, Paragominas, Rondon do Pará e Ulianópolis;

b) Imagens do sensor MODIS do satélite TerraClass, produto MOD13Q1 para período de 2004 a 2017;

c) Software MODIS Reprojection Tool (MRT);

d) Imagens dos sensores TM e OLI, dos satélites da série LANDSAT 5, 7 e 8 , orbitas/ponto 222/62, 222/63, 223/62 e 223/63 para os anos de 2004 a 2017;

e) Apache2 (Hypertext Preprocessor PHP 5);

f) Geoserver.

\subsection{Métodos}

\subsubsection{Polígonos de áreas embargadas}

Os polígonos de áreas embargadas foram obtidos a partir da lei de acesso a informação, no site do IBAMA, Serviço de Informação ao Cidadão SIC. Esta lei permite a qualquer cidadão consultar os órgãos públicos e acessar as informações de que necessita, desde que não sejam sigilosas. Dessa forma, o shapefile foi baixado no sistema compartilhado de informações ambientais (http://siscom.ibama.gov.br/; IBAMA, 2016).

Para o desenvolvimento deste estudo foram selecionadas apenas as áreas embargadas por desmatamento. Além disto, os seguintes filtros referentes aos autos de infração foram aplicados:

a) Polígonos situados nos quatro municípios estudados (Dom Eliseu, Paragominas, Rondon do Pará e Ulianópolis);

b) Embargos por: i) destruir; ii) desmatar; iii) danificar florestas ou qualquer tipo de vegetação nativa; iv) infração da flora nãoclassificada;

c) Período entre 2004 e 2016; 
d) Existência de vértices contornando a área embargada e fechando a poligonal.

\subsubsection{Imagens MODIS/TERRA}

As imagens MODIS utilizadas neste trabalho foram obtidas via File $\begin{array}{lllll}\text { Transfer } & \text { Protocol } & \text { (FTP) link }\end{array}$ (https://e4ft101.cr.usgs.gov/MOLT/MOD13Q1.005/). O produto selecionado foi o MOD13Q1 Índice de vegetação composição de 16 dias com 250 metros de resolução espacial (HUETE et al., 2002), tile h13v09, adquiridas em formato HDF para o período de janeiro de 2004 a outubro de 2016, o que perfaz aproximadamente 300 imagens.

O programa MRT foi usado para converter os dados HDF em GeoTIFF e para aplicar o sistema de projeção geográfica adequado para o trabalho.

\subsubsection{Imagens TM, OLI/LANDSAT 5 e 8}

As imagens dos sensores TM e OLI, dos satélites da série LANDSAT 5 e 8, foram baixadas do site do Earth Explorer da United States Geological Survey - USGS (https://earthexplorer.usgs.gov/), onde estas foram fornecidas ortorretificadas, dispensando o registro (TUCKER; GRANT; DYKSTRA, 2004). Foi levado em consideração, para a escolha das imagens, a cobertura de nuvens, que dependendo da sua intensidade pode prejudicar a análise das áreas de interesse. Foram selecionadas 52 imagens do site Earth Explorer, abrangendo um período de 13 anos. As buscas foram feitas pelas órbitas/ponto 222/62, 222/63, 223/62 e 223/63 que juntas abrangem a área dos 4 municípios. Após o download das imagens selecionadas, foi realizada composição colorida falsa cor na ordem de bandas R5G4B3 para as imagens do sensor TM e R6G5B4 para as do sensor OLI. 


\subsubsection{A aplicação web}

A Figura 2 mostra uma tela do sistema web desenvolvido. A interação com o usuário ocorre através de uma página web com interface intuitiva e de fácil entendimento, desenvolvida com base em Java Script e Plataforma PHP, usando a interface de visualização do sistema OpenStreetMap para geolocalização dos polígonos embargados (MORAES et al., 2017).

Ao arrastar o mouse no mapa, o usuário indica a região da poligonal que será utilizada, podendo após escolher sua área de interesse clicar dentro do polígono e então o usuário receberá como retorno visual um combobox, também conhecido como caixa de seleção, para selecionar a classe que deseja, após a verificação feita no gráfico da série temporal de índice de vegetação, como pode ser visto no item (a) da Figura 2. Simultaneamente é mostrado um gráfico com a série temporal na parte inferior da página. A informação dessa série temporal recuperada pelo clique na área selecionada refere-se a um pixel MODIS.

O item (b) da Figura 2 mostra as séries temporais NDVI de 14 anos (2004 a 2017) usando gráfico interativo. A linha vermelha representa as séries temporais NDVI do ponto selecionado dentro do polígono embargado. $\mathrm{O}$ eixo das abscissas representa o tempo medido em anos e o eixo das ordenadas indica valores de NDVI.

Como pode ser visto no item (b) da Figura 2, existem sequências de informações que correspondem a aproximadamente 18 anos do mesmo ponto (janeiro de 2000 a junho 2017), gerando uma grande quantidade de dados que tornaria muito custosa a extração manual desses índices. 
Figura 2 - Interface da página web

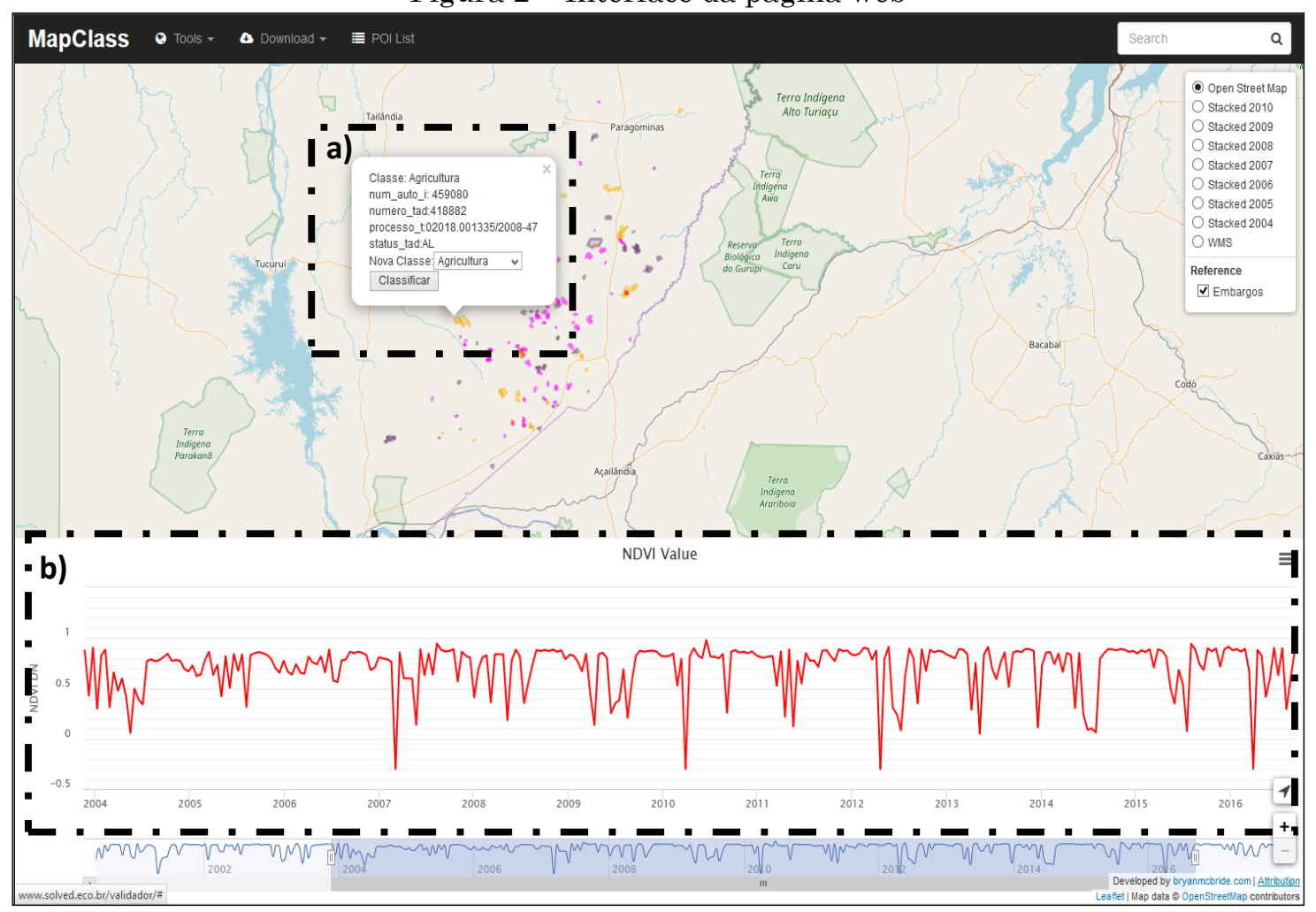

Fonte: Elaborada pelos autores.

\subsubsection{Analise dos polígonos de embargo}

As análises dos polígonos embargados foram realizadas na aplicação web onde se inseriram os dados oficiais de embargos para serem analisados individualmente. A classificação dos polígonos usando os perfis temporais dos índices de vegetação foram realizadas com base no conhecimento prévio dos padrões temporais dos principais objetos estudados dentro dos polígonos desmatados, que são pasto, agricultura e regeneração.

A Figura 3 apresenta um perfil do índice de vegetação para uma região analisada, mostrando valores altos de 2004 até meados de 2010 caracterizando floresta, após 2011 é possível perceber perturbações que alteram o padrão dos valores, mostrando que a área está em processo de desmatamento. Por fim, pode-se observar que a região selecionada está sendo usada para agricultura, tendo em vista sua variação com um perfil estreito e alta variação sazonal. 
Figura 3 - Perfil temporal do índice de vegetação

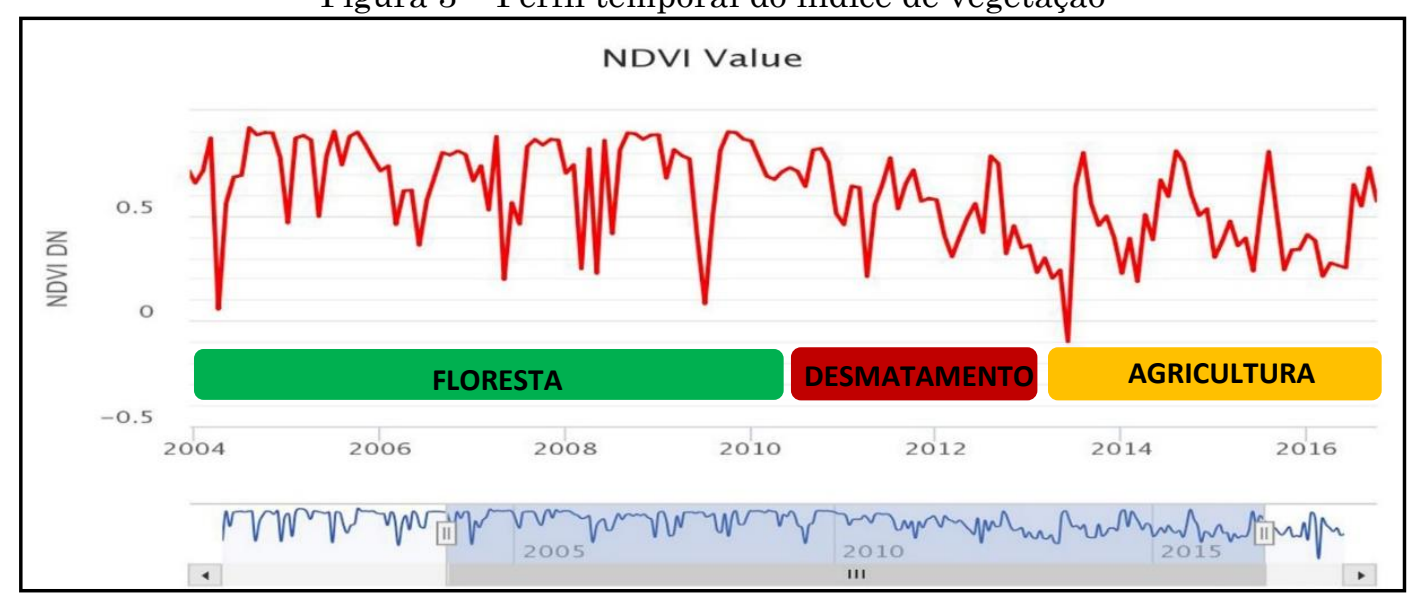

Fonte: Elaborada pelos autores.

Alguns estudos realizados anteriormente também foram consultados e serviram de base teórica como (GALFORD et al., 2008), (FREITAS et al., 2011), (ARVOR et al., 2011) e (ADAMI et al., 2012) que apresentam alguns exemplos do perfil temporal dos índices de vegetação de produtos MODIS para caracterizar mudanças de uso do solo, como pasto e agricultura (Figura 4).

Figura 4 - Série temporal de índice de vegetação para agricultura, pasto, cerrado e floresta

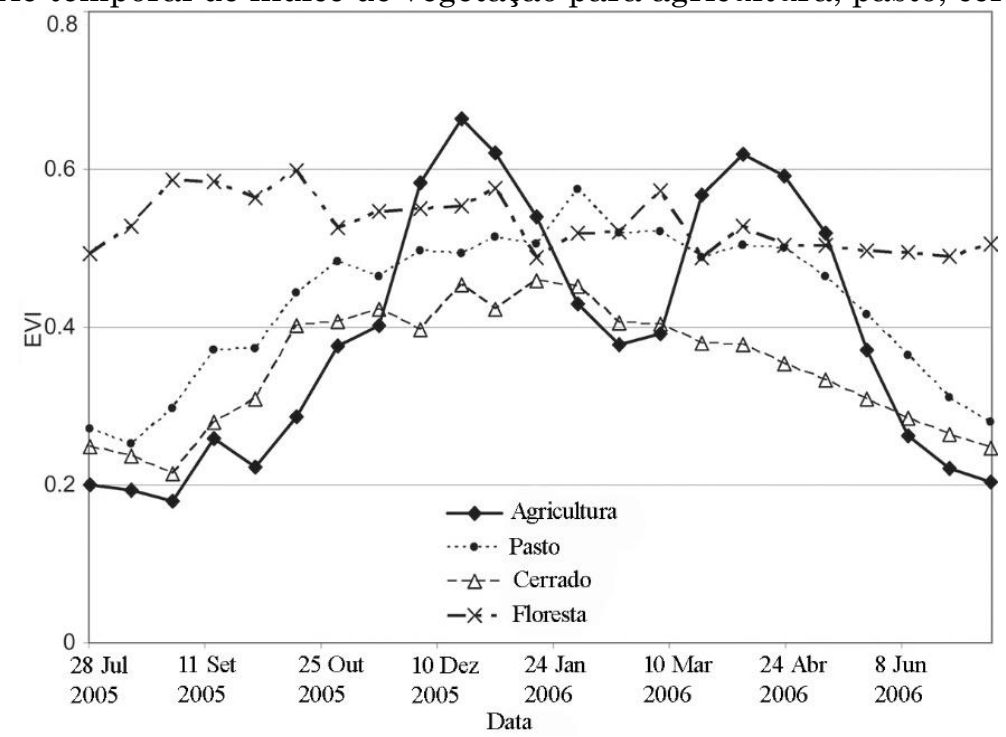

Fonte: adaptado de ARVOR et al., (2011).

\section{Resultados e Discussão}

Analisando as séries temporais na Figura 5, observa-se uma diminuição nos valores NDVI a partir de 2012, indicando uma perda 
significativa da biomassa devido ao processo de desmatamento. De 2012 a 2016, não houve crescimento de vegetação como indicado pelo baixo perfil NDVI durante este período. Risso et al. (2012) perceberam que para as áreas de pastagens os índices de vegetação apresentam uma tendência de valores menores, visto que a cobertura do solo, na maioria dos casos, não está completa, tendo assim uma heterogeneidade da resposta espectral. Portanto, a classe de pastagem é facilmente identificada no perfil temporal de índices de vegetação.

Figura 5 - Perfil espectro temporal do ponto selecionado (NDVI) NDVI Value

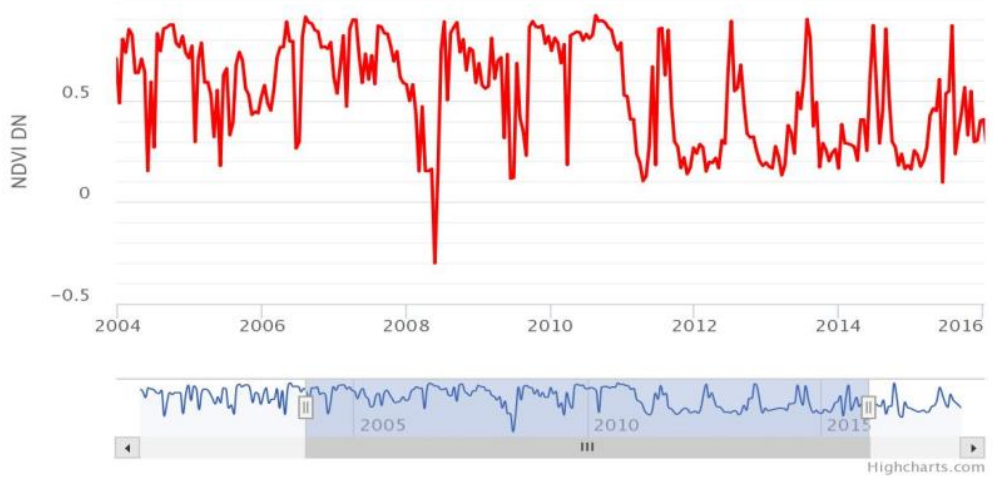

Fonte: Elaborada pelos autores.

A Figura 6 mostra uma região dentro do polígono de embargo analisado acima, no município de Rondon do Pará, que tinha sua área totalmente florestada no ano de 2004 (esquerda). E mostra a área do polígono que foi desmatada no ano de 2009 que não está em processo de regeneração e encontra-se a classe pasto no ano de 2017 (direita), validando a série temporal. 
Figura 6 - Imagem do satélite landsat-5, sensor TM, ano 2004 (esquerda) e Imagem do satélite landsat-8, sensor OLI, ano 2017 (direita). Município de Rondon do Pará. Polígono

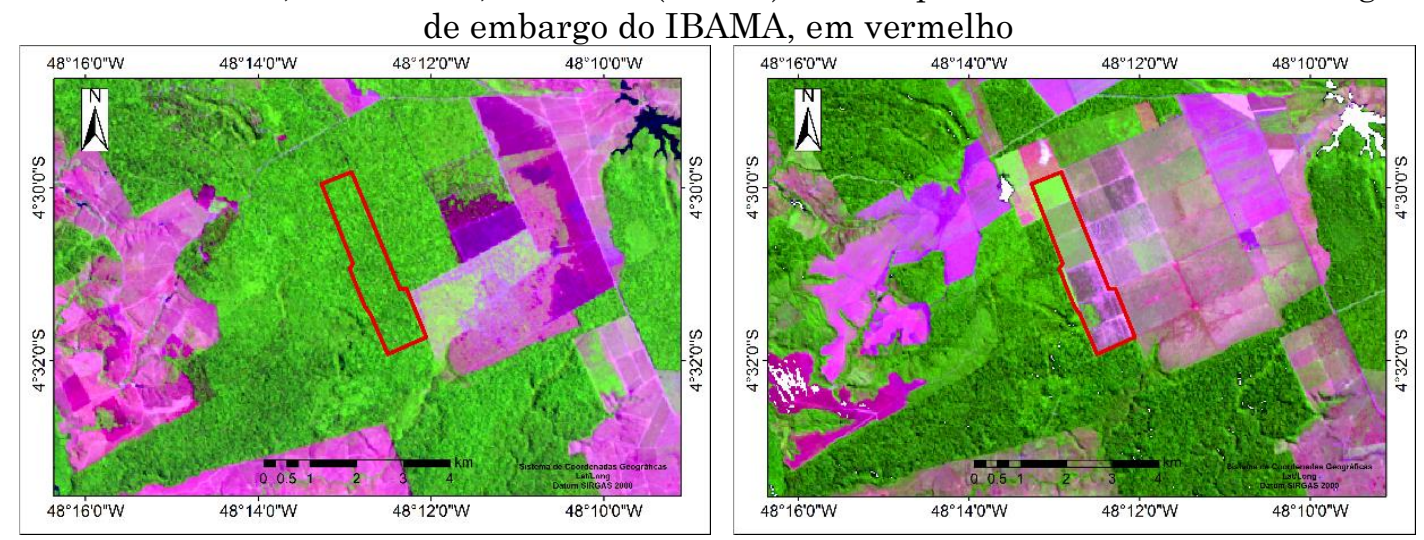

Fonte: Elaborada pelos autores.

Carmo (2014) apresentou padrões para definir áreas que estão sendo usadas para agriculturas, onde os perfis espectrais tendem a ter um rápido aumento nos valores durante o período de desenvolvimento da cultura, seguido por uma forte queda, criando um perfil mais estreito. Portanto, neste trabalho, áreas com alta variação sazonal, perfis estreitos e picos nos valores dos índices de vegetação inferiores a 0,7 foram classificados como agricultura.

A análise do perfil espectro-temporal da Figura 7 mostra a sazonalidade de um ponto selecionado dentro de um polígono de uma área que foi desmatada no final de 2004, autuada e embargada em 2007. A análise visual da série temporal indica uma perda da biomassa caracterizando desmatamento no final de 2004. E a partir de 2008 um rápido aumento seguido de uma rápida diminuição dos valores do índice de vegetação indicando ciclos de agricultura. Podendo também observar com esta análise, em qual trimestre o processo de desmatamento começou. 
Figura 7 - Perfil espectro temporal do ponto selecionado (NDVI) NDVI Value

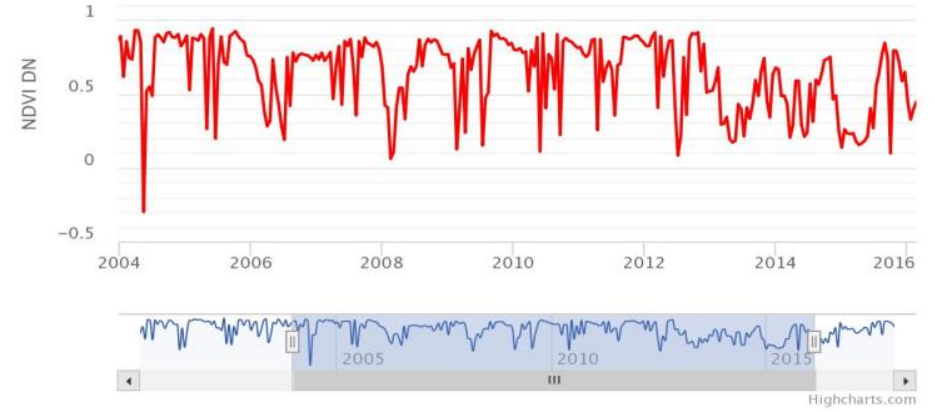

Fonte: Elaborada pelos autores.

A Figura 8 confirma o apresentado na análise da série temporal mostrando uma região florestada no município de Ulianópolis em 2004 (esquerda). E mostra a mesma com classe de agricultura no ano de 2017 (direita).

Figura 8 - Imagem Landsat-5, ano 2004 (esquerda). E imagem Landsat-8, ano 2017. Município de Paragominas. Polígono de embargo em amarelo
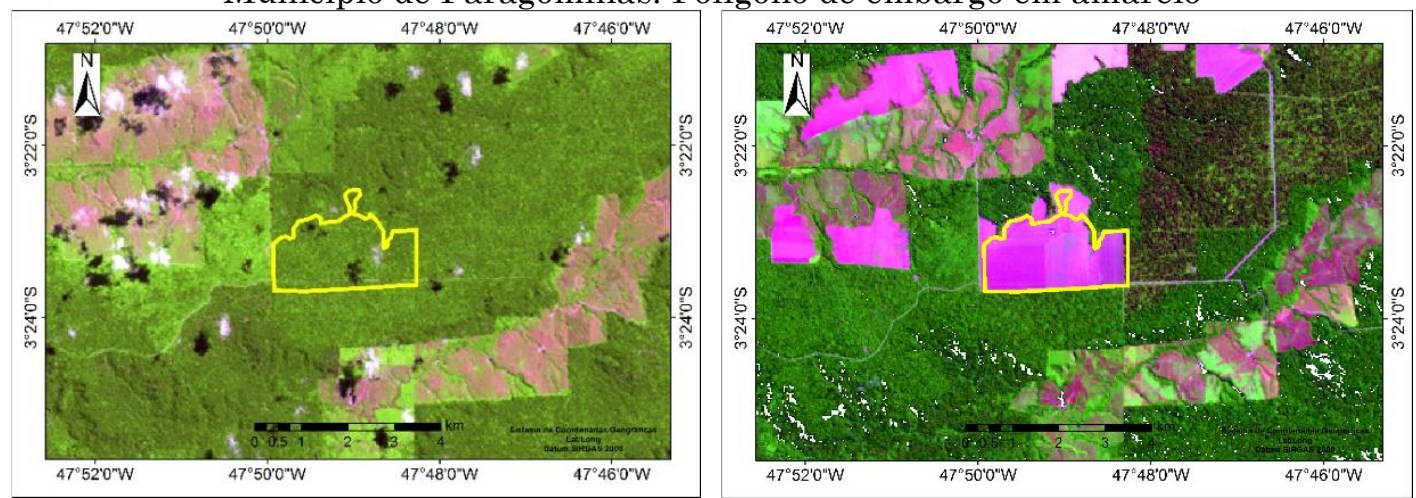

Fonte: Elaborada pelos autores.

Rosendo (2005), em seu estudo, analisou o comportamento dos gráficos de séries temporais de índice de vegetação para a classe regeneração e verificou que estes apresentam valores mais elevados, próximos ao 1 (um).

$\mathrm{Na}$ Figura 9 o gráfico mostra a sazonalidade de um ponto selecionado dentro de um polígono de uma área no município de Dom Eliseu, que foi desmatada no final de 2005. De 2007 a 2016, houve crescimento de vegetação como indicado pelos valores elevados no perfil NDVI. 
Figura 9 - Perfil espectro temporal do ponto selecionado (NDVI). No município de Dom Eliseu NDVI Value

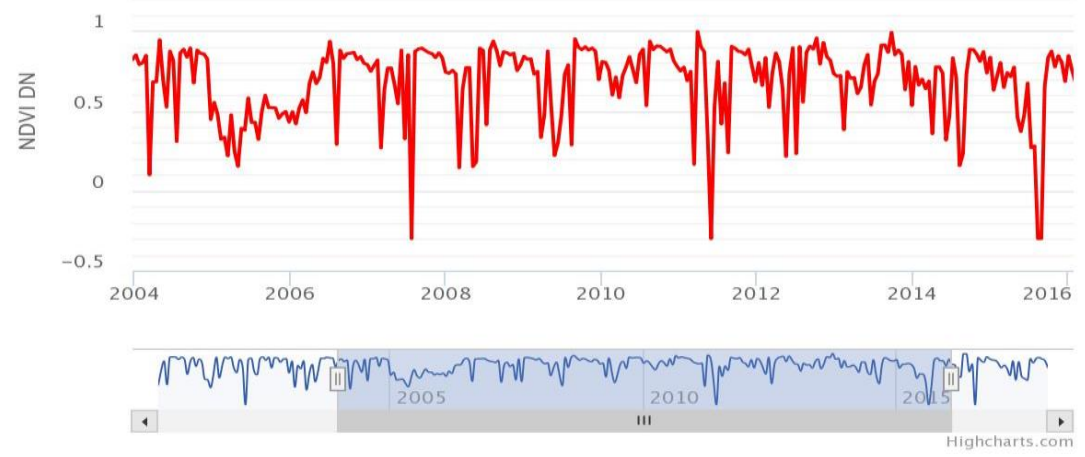

Fonte: Elaborada pelos autores.

A Figura 10 mostra a área do polígono no município de Dom Eliseu, em azul, desmatada em 2005 (esquerda). E a mesma área em processo de regeneração em 2017, validando a análise temporal (direita).

Figura 10 - Imagem Landsat-5, ano 2005 (esquerda). Imagem Landsat-8, ano 2017. Polígono de embargo em azul. Município de Dom Eliseu

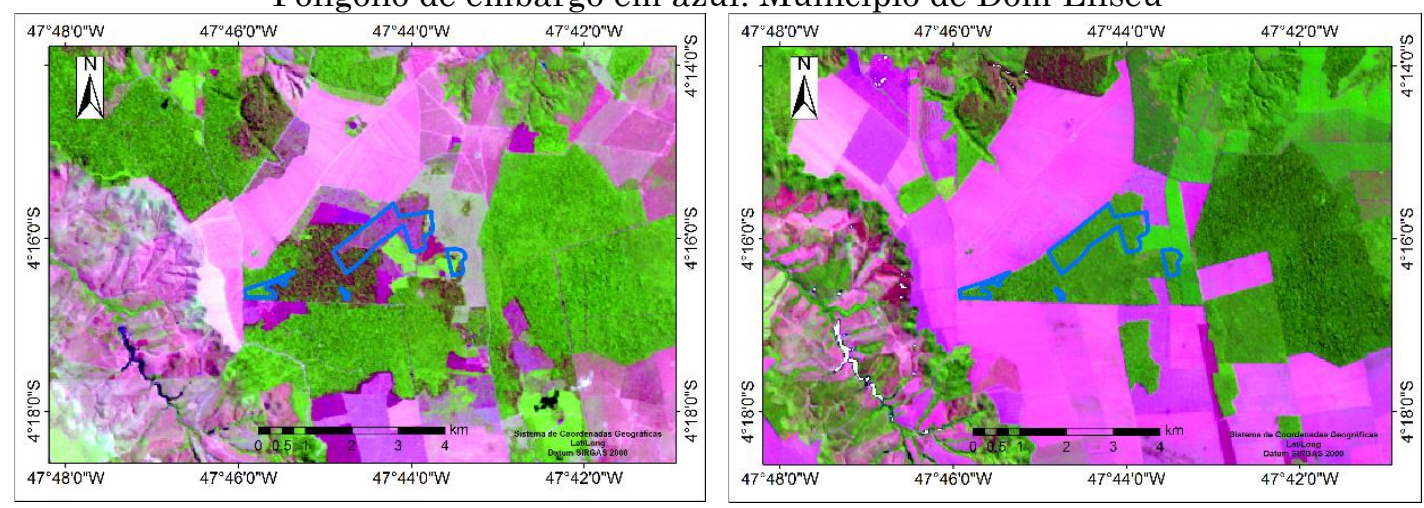

Fonte: Elaborada pelos autores.

Das 144 áreas de embargos analisados e classificados na aplicação foram encontrados 87 polígonos com a classe pasto, 14 polígonos com agricultura e 43 em processo de regeneração, de acordo com a distribuição do gráfico na Figura 11.

Rev. Bras. de Cartografia, vol. 70, Edição Especial "XXVII Congresso Brasileiro de 
Figura 11 - Distribuição geral das classes encontradas nos quatro municípios estudados do estado do Pará

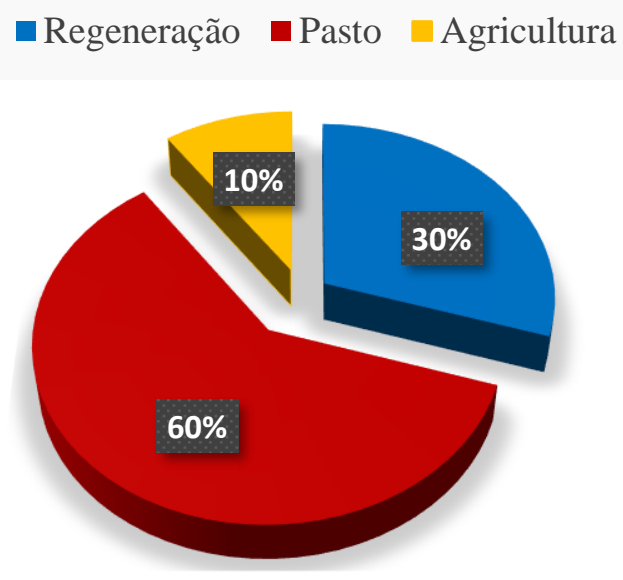

Fonte: Elaborada pelos autores.

\section{Conclusão}

Este estudo identificou que das 144 amostras de embargos de infrações ambientais selecionadas, em apenas $30 \%$ dos polígonos houve o cumprimento do embargo (regeneração natural), enquanto os outros $70 \%$ estavam irregulares, tendo sido encontradas classes de agricultura ou pasto, caracterizando descumprimento de embargo. Considera-se que o uso da ferramenta web integrada aos dados sensor MODIS mostrou-se adequada para a detecção do descumprimento de embargo e deteç̧ão do início do desmatamento nessas áreas, apresentando-se como excelente recurso de suporte para a fiscalização ambiental. Os resultados também indicam o elevado potencial das análises espectro-temporais de índices de vegetação para o monitoramento da cobertura vegetal, podendo-se identificar em qual trimestre teve início o processo de desmatamento. Cabe destacar que há questões relacionadas com a dimensão e forma do polígono que pode invalidar a análise do perfil temporal NDVI-MODIS. Para casos de polígonos estreitos ou com área menor que 25 ha recomenda-se o uso das imagens da série Landsat para garantir acuidade. 


\section{Referências}

ADAMI, M. Remote sensing time series to evaluate direct land use change of recent expanded sugarcane crop in Brazil. Sustainability, vol. 4, n. 4, 2012 pp. 574585.

ALMEIDA, C. A. High spatial resolution land use and land cover mapping of the Brazilian Legal Amazon in 2008 using Landsat-5/TM and MODIS. Acta Amazônica, vol. 46, n. 3, 2016 pp. 291-302

ANDERSON, L. O. Assessment of deforestation in near real time over the Brazilian Amazon using multitemporal fraction images derived from Terra MODIS. IEEE Geoscience and Remote Sensing Letters, vol. 2, n. 3, 2005 pp. 315318

ARVOR, D. Classification of MODIS EVI time series for crop mapping in the state of Mato Grosso, Brazil. International Journal of Remote Sensing, vol. 32, n. 22, 2011 pp. $7847-7871$

BECKER, B. Amazônia: desenvolvimento e soberania. In: Brasil: O Estado de uma nação. Rio de Janeiro: Instituto de Pesquisa Econômica e Aplicada IPEA. 2005, pp.199-250

BIZZO, E. Priorização de municípios para prevenção, monitoramento e controle de desmatamento na Amazônia: uma contribuição à avaliação do Plano de Ação para a Prevenção e Controle do Desmatamento na Amazônia Legal (PPCDAm). Desenvolvimento e Meio ambiente, vol. 42, 2017.

BRASIL. Decreto $\mathrm{n}^{0}$ 6.514, de 22 de julho de 2008. Dispõe sobre as infrações e sanções administrativas ao meio ambiente, estabelece o processo administrativo federal para apuração destas infrações, e dá outras providências. Brasília, Diário Oficial da União, 22 de julho de 2008.

BRASIL. Decreto $n^{\circ} 7.830$, de 17 de outubro de 2012. Dispõe sobre o Sistema de Cadastro Ambiental Rural, o Cadastro Ambiental Rural, estabelece normas de caráter geral aos Programas de Regularização Ambiental, de que trata a Lei no 12.651, de 25 de maio de 2012, e dá outras providências. Brasília, Diário Oficial da União, 17 de outubro de 2012.

BRASIL. Decreto ${ }^{\circ}$ 6.321, de 21 de dezembro de 2007. Dispõe sobre ações relativas à prevenção, monitoramento e controle de desmatamento no Bioma Amazônia, 
bem como altera e acresce dispositivos ao Decreto no 3.179, de 21 de setembro de 1999, que dispõe sobre a especificação das sanções aplicáveis às condutas e atividades lesivas ao meio ambiente, e dá outras providências. Brasília, Diário Oficial da União, 21 de dezembro de 2007.

BRASIL. Grupo Permanente de Trabalho Interministerial. Plano de Ação para a Prevenção e o Controle do Desmatamento na Amazônia Legal (PPCDAM). Brasília: Casa Civil, 2004.

CABRAL, I. P. de S. Novas ferramentas para monitoramento ambiental usando SIG Web. Tese de doutoramento. Universidade Federal do Rio Grande do Norte, Programa de Pós-Graduação em Engenharia Elétrica, Natal, 2008. $86 \mathrm{p}$.

CARMO, N. C. Mapeamento agrícola com imagens de sensores remotos orbitais para apoio ao gerenciamento de recursos hídricos. Dissertação de mestrado. Universidade Federal de Goiás, Programa de Pós-graduação em Engenharia do Meio Ambiente, 2014. 124p.

CASTELO, T. B. Brazilian forestry legislation and to combat deforestation government policies in the amazon (Brazilian amazon). Ambiente \& Sociedade, vol. 18, n. 4, 2015. pp. 221-242

DINIZ, C. G. DETER-B: the new Amazon near real-time deforestation detection system. IEEE Journal of Selected Topics in Applied Earth Observations and Remote Sensing, vol. 8, n. 7, 2015. pp.3619-3628

ESPÍNDOLA, C. E. A tecnologia da informação como meio para facilitar o acesso do cidadão aos serviços públicos. Anais do IV Congresso CONSAD de Gestão Pública, Brasília, 2011.

HUETE, A. Overview of the radiometric and biophysical performance of the MODIS vegetation indices. Remote Sensing of Environment, vol. 83, n. 1, 2002 pp. 195-213

INDICADORES DE DESENVOLVIMENTO SUSTENTÁVEL DO BRASIL 2015. Site <http://biblioteca.ibge.gov.br/visualizacao/livros/liv94254.pdf> acessado em fevereiro de 2017. IBGE, Instituto Brasileiro de Geografia e Estatística. Site <https://www.ibge.gov.br/> acessado fevereiro de 2017.

IPEA - AGROPECUÁRIA POR MUNICÍPIO 2010. Site $<$ http://www.ipeadata.gov.br/>, acessado em agosto de 2017. IPEA. Instituto de 
Pesquisa Econômica Aplicada. Site < http://www.ipea.gov.br/>, acessado em agosto de 2017.

IPEA, GIZ \& CEPAL. Avaliação do Plano de Ação para Prevenção e Controle do Desmatamento na Amazônia Legal - PPCDAm 2007 - 2011. 2011. 54p

KALAMANDEEN, M. Pervasive Rise of Small-scale Deforestation in Amazonia. Scientific reports. vol. 8, n. 1, 2018. pp. 1600

LIMANA, C. C. O Sensoriamento Remoto Como Ferramenta Didática na Educação Profissional e Tecnológica. 2014. Dissertação de mestrado. Universidade Federal de Santa Maria, Programa de Pós-Graduação em Geografia e Geociências, Santa Maria, 2014. 74p.

LUI, G. H. Ocupação humana e transformação das paisagens na Amazônia brasileira. Amazônica. Revista de antropologia, vol. 1, n. 1, 2016.

FREITAS, R. M. Virtual laboratory of remote sensing time series: visualization of MODIS EVI2 data set over South America. Journal of Computational Interdisciplinary Sciences, vol.2, n.1, 2011. pp.57-64

GALFORD, G. L. Wavelet analysis of MODIS time series to detect expansion and intensification of row-crop agriculture in Brazil. Remote sensing of environment, vol. 112 , n. 2 , 2008. pp. 576-587

MATOS, F. L. L. C.C. Análise das taxas anuais de desmatamento na Amazônia Legal a partir da relação entre autos de infração e área desmatada no período entre 2000 e 2014. 2016. Dissertação de mestrado. Universidade de Brasília, Programa Pós-Graduação em Geografia, Brasília, 2016. 90p.

MITTERMEIER, R. Uma breve história da conservação da biodiversidade no Brasil. Megadiversidade, vol. 1, n. 1, 2005. pp. 14-21.

PRODES - MONITORAMENTO DA FLORESTA AMAZÔNICA BRASILEIRA POR SATÉLITE. Site

<http://www.obt.inpe.br/OBT/assuntos/programas/amazonia/prodes>, acessado em setembro de 2017. Coordenação Geral de Observação da Terra INPE. <http://www.obt.inpe.br>, acessado em setembro de 2017.

MORAES, D. R. V. Aplicação Web para Monitoramento de Áreas Embargadas por Desmatamento, no Município de Dom Eliseu-PA. Anais do XXVII Congresso Brasileiro de Cartografia, Rio de Janeiro, 2017. pp. 879-883. 
NEVES, A.P.F.G. Correlação entre pecuária e desmatamento em municípios da mesorregião sudeste do estado do Pará, Brasil. AMBIÊNCIA, vol. 10, 2014. pp.795-806.

RISSO, J. Índices de vegetação MODIS aplicados na discriminação de áreas de soja. Pesquisa Agropecuária Brasileira. vol. 47, n. 9, 2012. pp. 1317-1326.

ROSENDO, J. dos S. Índices de vegetação e monitoramento do uso do solo e cobertura vegetal na bacia do Rio Araguari-MG-utilizando dados do sensor MODIS. 2005. 152 f. Dissertação de mestrado. Universidade Federal de Uberlândia, Programa de Pós-Graduação em Geografia, Uberlândia, 2005. $152 p$.

PANAZZOLO, A. P. Info ambiente: Gestão Ambiental de rodovias com SIG-WEB. Anais do Congresso Brasileiro de Gestão Ambiental, Belo Horizonte, 2014

PIONTEKOWSKI, V. J. Deforestation assessment in the state of Rondônia between 2001 and 2011. Floresta e Ambiente, vol. 21, n. 3, 2014. pp. 297-306

SANTOS, J. R. Integração de imagens LANDSAT/ETM+ e CBERS-2/CCD para deteç̧ão de mudanças em área da Amazônia sob domínio da floresta de transição. Revista Brasileira de Cartografia. Vol. 1 n. 57, 2005. pp.15-21

SILVA, J. A. A. O Código Florestal e a Ciência. $1^{\mathrm{a}}$ ed. São Paulo: Sociedade Brasileira para o Progresso da Ciência, 2011. 124p.

THERY, H. Situações da Amazônia no Brasil e no continente. Estudos Avançados, vol. 19 , n. 53, 2005. pp. 37

TUCKER, C. J. NASA's Global Orthorectified Landsat Data Set. Photogrammetric Engineering \& Remote Sensing. vol.70, n. 3, 2004. pp.313-322

VALERIANO, D. M. Dimensões do Desmatamento na Amazônia Brasileira. População e Sustentabilidade na era das Mudanças Ambientais Globais: Contribuições para uma Agenda Brasileira, 2012. pp223-238.

VEIGA, J. E. Diversidade biológica e dinamismo econômico no meio rural. In: MAY, Peter (org.) Economia do meio ambiente: teoria e prática. 2. ed. Rio de Janeiro: Campus, 2010. pp. 289-308 